\title{
Developmental Changes in Map-reading Skills
}

\author{
Neil Bluestein \\ Beersheva, Israel \\ Linda Acredolo \\ University of California, Davis
}

\begin{abstract}
Bluestein, Neil, and Acredolo, Linda. Developmental Changes in Map-reading Skills. Crimld DeVElopment, 1979, 50, 691-697. The ability of 3-, 4-, and 5-year-old children to infer the position of an object in a room from information contained on a map was assessed under 5 conditions: $(a)$ map aligned inside the room (the pretest); $(b)$ map aligned outside the room; $(c)$ map rotated $180^{\circ}$ inside the room; $(d)$ map rotated $180^{\circ}$ outside the room; and $(e)$ map held vertically outside the room. The results indicated that children as young as 3 years could read a map aligned inside a room but that the ability to compensate for a rotated map did not develop until age 5. The position of the map inside or outside the room did not affect performance in either the aligned or rotated conditions, with the exception that fewer egocentric errors were made in the rotated-outside condition than in the rotated-inside condition. The vertically held map was easily interpreted by most children who succeeded on the pretest, thus indicating that "up" and "down" are readily interchanged with "near" and "far." All the results are discussed in relation to a 2-component model of map reading.
\end{abstract}

As an activity requiring symbols to substitute for, to "represent," sets of concrete objects and relations among objects in the world, the act of using a map is a prime example of the symbolic or semiotic function at work. More particularly, information about mapping skills may help clarify the results of developmental studies in which the drawing of sketch maps or the building of models has been relied on to assess internal cognitive representations of space (e.g., Ladd 1970; Siegel \& Schadler 1977; Pick, Acredolo, \& Gronseth, Note 1). In these studies the ability to deal with maps is assumed, and any failures in the resulting maps are attributed to inadequacies in the underlying representation, when in reality the problem may be in the translation of that representation or the space itself to the two- or three-dimensional model. The present experiment was designed to provide some initial data on one aspect of this phenomenon: map reading.

For the purposes of the present discussion, map reading is defined as the ability to make judgments about position in a three-dimensional, large-scale space from information presented in a two- or three-dimensional smallscale representation of the space. Such mapreading inferences appear to be based on at least two distinct cognitive processes, each of which may prove to have a separate developmental history. The first is the differentiation of cartographic or pictographic symbols and the understanding that they refer to real three-dimensional counterparts. Blaut, McCleary, and Blaut (1970) refer to this as the "semantic interpretation" component. Clearly, the development of this component will vary with the degree of abstractness of the cartographic symbols and with the amount of detail presented in the map.

The second process is the projection or superimposition of the map upon the space, or the space upon the map, so that judgments about position can be made from one to the other. This latter achievement is no simple accomplishment. In fact, it seems itself to have several components. First, the child must recognize that the relationships among the cartographic symbols on the map represent the relationships among the actual objects in space. However, this act of relating map and space does not guarantee that correct judgments will be made. The child must also recognize the possibility that the map and the space may not be perfectly aligned and, if not, compensate for the nonalignment. For example, the map

A portion of this study was presented at the biennial meeting of the Society for Research in Child Development, New Orleans, March 1977. Requests for reprints should be sent to Linda P. Acredolo, Department of Psychology, University of California, Davis, California 95616.

[Child Development, 1979, 50, 691-697. (C) 1979 by the Society for Research in Child Development, Inc. $0009-3920 / 79 / 5003-0009 \$ 00.75]$ 
reader must often transform a vertical view of the map to the horizontal perspective from which the surrounding environment is usually viewed, while at the same time preserving the integrity of the features and the spatial relations among them. He or she should also be able to compensate for rotation of the map relative to the space. Finally, under the special condition where the map and the space are not viewed simultaneously, memory factors may also play a role.

Surprisingly little is known about the development of these abilities beyond the fact that it takes time to learn cartographic symbols and conventions, the first of the three components discussed above (Rushdoony 1968). One of the few studies that has addressed these issues at all required 5-, 6-, and 7-year-old children to identify features such as houses, roads, cars, and trees on aerial photographs of a community and make tracings of the photograph and solve navigational problems by indicating appropriate routes between features (Blaut et al. 1970). The authors concluded from the success of even the youngest children that "preliterate children of school-entering age can ... engage in a real, if primitive, form of map reading, map making, and map use" (p. 346). However, although the children did recognize features, and act similar to the first component of our model, the question remains whether their behavior actually constitutes evidence of the second component, the superimposition of the map and the actual space. It seemed clear to us that it did not.

Some indirect information about this second component is provided in studies requiring children to duplicate on their own model space the location of a target object placed on the experimenter's model space (e.g., Piaget \& Inhelder 1967; Pufall \& Shaw 1973). While these studies do not involve inferences to large-scale spaces, they have revealed that the preoperational child often codes location egocentrically when one space is rotated $180^{\circ}$ relative to the other. Still to be determined, however, is whether this egocentrism is a pervasive predisposition which at this age influences any space-to-space inference, or whether it is simply a by-product of the particular paradigm employed to study mapping. After all, it is certainly true that many studies of spatial perspective taking have failed to find high proportions of egocentric responding once traditional procedures are altered (e.g., Borke 1975). In the studies in question here, the two spaces are traditionally located adjacent to one another, presumably eliminating the possible confounding effects of memory. Such visual proximity, however, may actually promote the relating of the two without examination of their alignment or nonalignment. In other words, a possible by-product of the juxtaposition of the maps may be the creation of a compelling perceptual array which lures the preoperational child into ignoring rotation in favor of replicating the visual array from his or her own perspective. Support for such a hypothesis comes both from studies of spatial perception itself (McKinney 1964) and from Piaget, who has repeatedly concluded that children during this stage of development have trouble dissociating their responses from the perceptual aspects of a situation. It follows, then, that the children's performances on mapping tasks might actually improve if the space and the map were not in close proximity to one another.

In response to the major issues discussed above, the present study was designed $(a)$ to augment the Blaut et al. (1970) study by providing infcrmation about the development of children's ability to make position judgments based on information from a simple two-dimensional map of a three-dimensional, large-scale space; and $(b)$ to test the hypothesis that egocentrism in maplike tasks is a result of the preoperational child's tendency to be influenced by the visual array presented by map and space in close proximity to one another.

\section{Method}

\section{Subjects}

The original sample of subjects included 60 children ( 22 3-year-olds, 28 4-year-olds, and 10 5-year-olds) from two middle-class suburban nursery schools. Of this original sample 46 passed a pretest, to be described below, and were allowed to complete the experiment. These 46 children included 123 -year-olds (six males and six females), 24 4-year-olds (12 males and 12 females), and 10 5-year-olds (four males and six females).

\section{Apparatus}

The major piece of apparatus consisted of a $12 \times 12$-foot $(3.66 \times 3.66 \mathrm{~m})$ collapsible room designed to enable control of the number and the saliency of the spatial cues available to the child. The room consisted of a wooden frame and four opaque curtains of white vinyl. A ceiling of white oilcloth allowed diffused light to enter. Four green boxes, $8 \times 8 \times 12$ 
inches $(.2 \times .2 \times .3 \mathrm{~m})$ in size, were positioned on the floor at the center of each wall. One corner of the curtains was open to function as a door; the other three corners were differentiated by a red box and two polyurethane foam shapes of different colors. There were two tables. One was positioned inside the room and surrounded by four chairs, each with its back to one of the walls. The other was positioned outside of the room about 6 feet $(1.83 \mathrm{~m})$ from the door. It had two chairs, one for the child and one for the experimenter (see fig. 1). Finally, materials included a small toy elephant about 4 inches $(.1 \mathrm{~m})$ in length and a twodimensional schematic representation of the room on a $12 \times 12$-inch $(.3 \times .3 \mathrm{~m})$ piece of cardboard. This map contained every distinctive feature of the room represented by colored line drawings from a vertical rather than aerial perspective, so that some pictorally represented three-dimensional information was provided to aid identification of the objects. The map also contained a separate representation of the toy elephant, with an adhesive backing, that could be repositioned on the map.

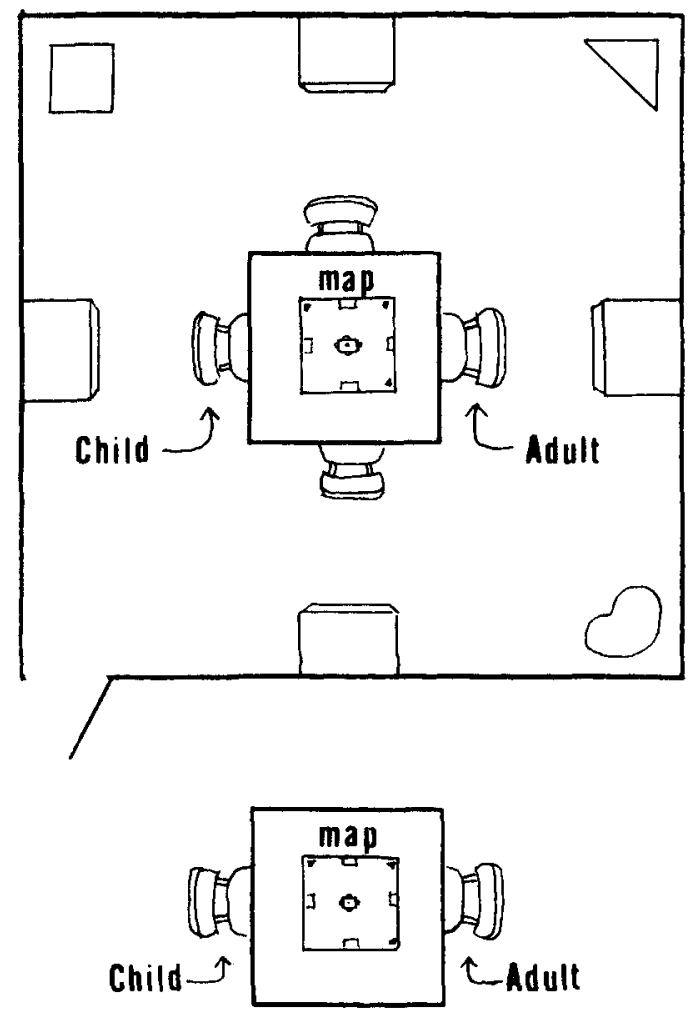

Fic. 1.-Experimental space and equipment

\section{Procedure}

For each subject the session began with a familiarization phase in which the child was seated at the inside table with the map and room perfectly aligned and asked to identify the pictures on the map and point to their referents. The experimenter then explained that the child was to use the pictures to find Peanut the elephant. In the conditions in which the map was located inside the room, the child was asked to stand outside momentarily while the experimenter hid the elephant and designated the position on the map. When the experimenter said "ready," the child returned to his or her seat in the room, viewed the map flat on the table, and went to find the elephant. In the conditions in which the map was placed outside the room, the experimenter entered the room without the child in order to hide the elephant and returned to designate its position on the map. With one exception, the child viewed the map flat on the table while seated and then went inside to retrieve the elephant. Once the child entered the room, he or she was not allowed to return to the map. In all cases the child was warned that the map might not be perfectly aligned with the room and to be very careful to choose the correct box. These basic procedures were repeated three times for all five conditions, a total of 15 trials per child.

Conditions.-The first condition each child experienced was the $0^{\circ}$-inside condition in which the map was placed on the inside table and perfectly aligned with the room. This condition served as a pretest since it was assumed that a child who was unable to infer the correct location from the map under these simple circumstances would be incapable of establishing a map-to-room association in the more complicated conditions to come. Subjects failing this condition were not tested further. In the $0^{\circ}$-outside condition, the map was positioned on the outside table but still aligned with the room. In other words, the child's perspective of the map was the same as his or her perspective upon entering the room. In this case, due to the removal of the map from the room itself, direct perceptual matching was not possible, and the child was forced to rely on either his memory of the map or of the space. In the $180^{\circ}$-outside condition, the map was positioned on the outside table and also rotated $180^{\circ}$ relative to the room. Consequently, in this condition the child was required not only to retain information about the map and/or space in memory but also to compensate for the non- 
alignment of the two. In the fourth condition, the $180^{\circ}$-inside condition, the map was again positioned on the inside table but was rotated $180^{\circ}$ relative to the room. In this case the presence of the map in the room enabled the child to forgo reliance on memory, but did require the child to compensate for the rotation. Obviously, a child who shortcuts this compensation by resorting to direct isomorphic matching in this condition will end up choosing the incorrect box directly opposite the correct one, an egocentric choice. Finally, the fifth condition, the vertical-outside condition, involved having the child view the map while seated at the table outside the room with the experimenter holding the map vertically by one corner to eliminate any horizontal alignment with the room. The corner held at the top was the corner diagonally opposite the door; consequently, the child's view of the map, disregarding the planar differences, could be interpreted as a close approximation of his or her view of the room upon entering (essentially a third $0^{\circ}$-aligned condition). This condition was included in order to determine at what point in the development of map reading a child acquires the flexibility necessary to perceive information presented in the vertical plane as isomorphic with spatial relations experienced in the horizontal plane. In other words, at what point does "up" become synonymous with "far away" and "down" with "close"?

Order.-Since all subjects were tested in all conditions, it was necessary to counterbalance the order in which the conditions were experienced in order to control for practice effects. This was done with two exceptions: the $0^{\circ}$-inside condition, because of its role as a pretest, was experienced first by all subjects, and the vertical-outside condition was experienced last by all subjects. Subjects were randomly assigned to the six possible orders of the remaining three conditions.

\section{Results}

In each trial of each condition, a subject had a choice of one of four boxes and, consequently, one chance in four to pick the correct box by choosing randomly. To decrease the likelihood that a child's success was due to such chance guessing, the subject, to be scored successful, was required to find the elephant correctly on not just one but two of the three trials in a given condition.

Table 1 presents the proportions of subjects at each age who scored successfully in

\section{TABLE 1}

Proportion of Subjects at Three Ages

Performing Successfully in Each of

Five Experimental Conditions

\begin{tabular}{lccccc}
\hline \hline & \multicolumn{5}{c}{ Condition } \\
\cline { 2 - 6 } $\begin{array}{c}\text { AGE } \\
\text { (Years) }\end{array}$ & $\begin{array}{c}0^{\circ} \\
\text { Inside }\end{array}$ & $\begin{array}{c}0^{\circ} \\
\text { Outside }\end{array}$ & $\begin{array}{c}180^{\circ} \\
\text { Inside }\end{array}$ & $\begin{array}{c}180^{\circ} \\
\text { Outside }\end{array}$ & $\begin{array}{c}\text { Vertical- } \\
\text { Outside }\end{array}$ \\
\hline $3 \ldots \ldots$ & .55 & .75 & .08 & .00 & .67 \\
$4 \ldots \ldots$ & .86 & .92 & .25 & .25 & .77 \\
$5 \ldots \ldots$ & 1.00 & 1.00 & .80 & .90 & .80 \\
\hline
\end{tabular}

Note. - Subjects not performing correctly in the $\theta^{\circ}$-inside condition were not tested in the remaining conditions and are therefore not included in the remaining columns.

each of the five conditions. As can be seen from the table, several dramatic age changes occurred. First, in the simplest condition of all, the $0^{\circ}$-inside condition which functioned as a pretest, analyses revealed that significantly fewer 3-year-olds performed successfully than either 4 -year-olds, $\chi^{2}(1)=4.49, p<.025$, or 5-yearolds (Fisher exact probability test, $p=.01$ ). In other words, almost half of the original group of 3-year-olds, compared with none of the 5year-olds, failed to pass the pretest and were eliminated from further testing. Second, the relatively high proportion of successful subjects (of those remaining after the pretest) at all ages in the $0^{\circ}$-outside condition indicates that if a subject could find the elephant when the map was aligned inside the room he or she could probably also do so when the map was aligned outside the room. There were no significant age differences for this condition. Third, in both the rotation conditions, $180^{\circ}$-outside and $180^{\circ}$-inside, the 5-year-olds performed significantly better than either of the two younger groups (Fisher exact probability tests, $p<.005$ in all four cases). The 3- and 4-year-olds, however, did not differ from one another. Finally, the vertical-outside condition produced relatively high proportions of success at all ages and resulted in no significant age differences.

Data analyses were also conducted to determine whether some conditions were significantly more difficult than others at any of the three ages. Again, the pattern of differences is clear from table 1. For the 3-year-olds, the $0^{\circ}$-outside condition resulted in a significantly larger proportion of successful responders than either the $180^{\circ}$-outside condition (McNemar test, $\left.\mathrm{x}^{2}[1]=7.1, p<.005\right)$ or the $180^{\circ}$-inside condition (McNemar test, $\chi^{2}[1]=4.9, p<$ $.025)$. Performances in the latter two conditions did not differ significantly. The supremacy of 
the $0^{\circ}$-outside condition over the $180^{\circ}$ rotation conditions was also found for the 4-year-olds (180 $0^{\circ}$-outside: McNemar test, $\chi^{2}[1]=14.06$, $p<.0005 ; 180^{\circ}$-inside: McNemar test, $\chi^{2}[1]$ $=13.07, p<.0005)$. The 5-year-olds, in contrast, performed with equal ease under both rotated and nonrotated conditions. The same pattern of results was found in comparing the vertical-outside condition with the two $180^{\circ}$ rotation conditions. For both the 3-year-olds and 4-year-olds, the vertical-outside condition was significantly easier than both the $180^{\circ}$-outside condition (3-year-olds: McNemar test, $\chi^{2}[1]=$ $4.2, p<.05$; 4-year-olds: McNemar test, $\chi^{2}[1]$ $=7.69, p<.01)$ and the $180^{\circ}$-inside condition (3-year-olds: McNemar test, $\chi^{2}[1]=3.2, p<$ .05 ; 4-year-olds: McNemar test, $\chi^{2}[1]=5.06$, $p<.025)$. Once again there were no differences between these conditions for the 5 -yearolds. There was also no difference between the $0^{\circ}$-outside condition and the vertical-outside condition for any of the age groups.

The question arises whether the fact that the vertical-outside condition was the last condition experienced accounts for its high proportion of successes. In order to determine if this were true, performances for the three conditions, whose order was counterbalanced, were examined $\left(0^{\circ}\right.$-outside, $180^{\circ}$-outside, and $180^{\circ}$ inside). The number of subjects performing successfully on the first condition experienced (whichever one that was) was compared with the number performing successfully on the second and third conditions experienced, since, presumably, practice effects will increase the number of correct responders across succeeding trials. No increase in the number of successes with succeeding trials was found. Indeed, the numbers are nearly identical for each trial. The absence of any practice effect here provides comfortable assurance that such effects alone probably cannot account for the large proportion of successes in the vertical-outside condition. The condition appears simply to be an easier map-reading situation for the 3 - and 4 -year-olds than the two $180^{\circ}$ rotation conditions.

Finally, the errors that the children made in the two rotation conditions were analyzed to determine what proportion were egocentric in nature. An egocentric error was defined to be the choice of the box directly opposite the correct box, a mistake which would automatically result from the child's ignoring the rotation of the map relative to the room. The analysis revealed that of the 30 unsuccessful subjects in the $180^{\circ}$-inside condition, 28 (or 93\%) had made egocentric errors. In contrast, among the 31 unsuccessful subjects in the $180^{\circ}$-outside condition, only 20 (or 65\%) made egocentric errors. A $\chi^{2}$ test indicated that this difference between the conditions was significant, $\chi^{2}(1)$ $=5.93, p<.01$. In other words, there was a significantly greater likelihood of egocentric errors when the map was located inside the room. With the map outside the room, a nearly identical number of errors occurred, but these were not as often egocentric in nature. $A$ withinsubject analysis revealed that, of the 31 children who made egocentric errors in these conditions, 17 (55\%) made egocentric errors in both, 11 (35\%) made an egocentric error inside but not outside, and only three (10\%) made an egocentric error outside but not inside.

\section{Discussion}

In general the results are consistent with an analysis of map reading as divisible into two components. The first, the ability to differentiate and interpret cartographic symbols as representative of objects in space was demonstrated to be present in all the children. In contrast, the second component, the ability to superimpose a map on a space, appeared to undergo a number of changes across the age span studied, starting with its absence among half the 3-year-olds, as indicated by their inability to locate the elephant in the $0^{\circ}$-inside condition even though all the cartographic symbols had been correctly identified. Only a few 4-year-olds and no 5-year-olds failed this condition. These results support the conclusion of Blaut et al. (1970) that even young children are capable of rudimentary map reading, but these results extend the age downward from 5 to 3 years and do so using a situation much closer to true map reading. Blaut et al. appear to have underestimated rather than overestimated the mapping skills of young children.

Although half the 3-year-olds and most of the 4-year-olds could make inferences from the map to the space, their accuracy was greatly influenced by the alignment of the map relative to the space. Unlike the 5-year-olds, both these younger groups failed to compensate for rotation of the map in the two $180^{\circ}$ rotation conditions. They chose the box directly across from the correct one in most cases, thus demonstrating the same type of egocentrism found at these ages by researchers using model spaces (e.g., Pufall \& Shaw 1973). The decline of 
such rotation errors between ages 4 and 5 in the present study is also remarkably consistent with the results of Pufall and Shaw, who found 4-year-olds making many more errors than 6year-olds in positioning an object on a model space after the experimenter's model with a similar object had been rotated $180^{\circ}$. Their conclusion was that it was the presence of discriminable features (topographical coding) in the space that enabled the older children to multiply the necessary relations. In the present study the spaces, both map and room, were topographically coded. The corners of both held different objects much as the four quadrants of the models of Pufall and Shaw were differentiated by different central forms and their corners by pegs of different colors. Consequently, the children had available to them simple relations like "between the door and the red box" to help them cope with the rotation. The 5-year-olds, like the 6-year-olds in the earlier study, took advantage of this information, but the 4-year-olds in both studies did not.

Although unable to compensate for $180^{\circ}$ rotation, the younger children in the present study were able to cope with the nonalignment of the vertical display of the map. Information presented in the vertical plane was easily interpreted as representative of relations in the horizontal plane, indicating that notions of near and far are readily interchangeable with up and down at these young ages, at least with simple maps and spaces. Still unanswered, however, is the question of whether a self-referential system is central to the child's analysis in the case of this transformation of perspectives. Would "egocentric" responses result from a presentation of the map held vertically but upside down? Some indication that they might is provided by Davol and Hastings (1967), who did find "egocentric" responding with rotated vertical stimuli. However, in that study both the experimenter's and the subject's displays were presented in the vertical plane, and consequently no vertical to horizontal transformation, as in the present study, was necessary.

Finally, removal of the map from the room did not affect accuracy at any age. Generally, what they could do inside they could do outside, and what they could not do inside they could not do outside either. Apparently, children as young as 3 have the capacity to remember position information viewed briefly and in representational form. Although accuracy was not affected, the type of error predominating was, the $180^{\circ}$-outside condition resulting in significantly fewer egocentric errors than the $180^{\circ}$-inside condition. One reason may be that, unlike the inside condition, the outside condition involved an initial diagonal view of the space through the corner door. Consequently, application of an egocentric frame of reference based on the relation of map to self established at the outside table may have been more difficult to apply. This lowered rate of egocentrism in the outside condition is also consistent with the results of Hardwick, McIntyre, and Pick (1976), who found that shielding 6-year-olds from viewing a room decreased egocentric responses to the task of specifying the position of objects after an imaginary rotation of the room. Together, these studies provide support for the hypothesis that spatial egocentrism may be at least partially a result of the child's being influenced by the immediate perceptual array. However, these studies also indicate that freeing the child from an egocentric perspective does not automatically result in accurate mental rotation. A child can know what the correct answer is not, without knowing what the correct answer is.

Although the children in the present study did demonstrate map-reading abilities, the task of learning to read maps is not finished at 5 years of age. Clearly the complexity of the map, the level of abstraction of the cartographic symbols, the "legibility" of the space depicted, the amount of area involved, the degree of nonalignment, and many other factors will all play important roles. In fact, each probably has its own developmental history, the detailing of which awaits future research. The purpose of the present study was to investigate map-reading skills in their most rudimentary form in order to provide a data base for these future investigations.

\section{Reference Note}

1. Pick, H. L.; Acredolo, L. P.; \& Gronseth, M. Children's knowledge of the spatial layout of their homes. Paper presented at the biennial meeting of the Society for Research in Child Development, Philadelphia, March 1973.

\section{References}

Blaut, J. M.; McCleary, G. F.; \& Blaut, A. S. Environmental mapping in young children. Environment and Behavior, 1970, 2, 335-349.

Borke, H. Piaget's mountains revisited: changes in 
the egocentric landscape. Developmental Psychology, 1975, 11, 240-243.

Davol, S., \& Hastings, M. L. Effects of sex, age, reading ability, SES, and display position on a measure of spatial relations in children. Perceptual and Motor Skills, 1967, 24, 375-387.

Hardwick, D. A.; McIntyre, C. W.; \& Pick, H. L., Jr. The content and manipulation of cognitive maps in children and adults. Monographs of the Society for Research in Child Development, 1976, 41(3, Serial No. 166).

Ladd, F. C. Black youths view their environment: neighborhood maps. Environment and Behavior, 1970, 2, 64-79.
McKinney, J. P. Hand schema in children. Psychonomic Science, 1964, 1, 99-100.

Piaget, J., \& Inhelder, B. The child's conception of space. New York: Norton, 1967.

Pufall, P., \& Shaw, R. Analysis of the development of children's spatial reference systems. Cognitive Psychology, 1973, 5, 151-175.

Rushdoony, H. A. A child's ability to read maps: summary of the research. Journal of Geography, 1968, 67, 213-222.

Siegel, A. W., \& Schadler, M. The development of young children's spatial representations of their classrooms. Child Development, 1977, 48, 388394. 
This document is a scanned copy of a printed document. No warranty is given about the accuracy of the copy. Users should refer to the original published version of the material. 